\title{
Das Modul Werbeslogans. Eine korpusinformierte lexikografische Ressource zum aktuellen Gebrauch von Werbeslogans außerhalb der Domäne Werbung
}

Janja Polajnar, Department of German, Faculty of Arts, University of Ljubljana, Ljubljana, Slovenia (janja.polajnar@ff.uni-lj.si)

Zusammenfassung: Der vorliegende Artikel setzt sich zum Ziel, das Modul Werbeslogans, das in das OWID-Sprichwörterbuch am Leibniz-Institut für Deutsche Sprache nachhaltig integriert ist, in seiner Entstehung darzustellen. Es handelt sich um eine korpusinformierte und nach Kriterien der wissenschaftlichen Lexikografie erarbeitete Onlinedokumentation von Werbeslogans bzw. von aktuell gebräuchlichen verfestigten Sätzen aus der Werbung, die bereits Einzug in die Gemeinsprache gefunden haben. Da diese Slogans ähnlich wie Sprichwörter gebraucht werden, wurden sie auch ähnlich beschrieben. Das Modul basiert auf den innovativen lexikografischen Konzepten im Sprichwortbereich, die im EU-Projekt SprichWort. Eine Internetplattform für das Sprachenlernen für die Sprichwort-Plattform entwickelt wurden, und auf dem OWID-Sprichwörterbuch; es ist innovativ und stellt keine Fortschreibung tradierter Wörterbücher im Bereich der Phraseologie dar. Im Artikel wird einerseits die korpusinformierte Methodik zur Analyse des Slogangebrauchs außerhalb der Domäne Werbung expliziert und andererseits der lexikografische Prozess, die Konzeption der Mikrostruktur der Slogan-Artikel sowie die verschiedenen Zugriffsmöglichkeiten in OWID (OnlineWortschatz-Informationssystem Deutsch) dargestellt. Die dargestellte lexikographische Behandlung von Werbeslogans im Rahmen von OWID ist (in der germanistischen Lexikographie) ein Novum und trägt zur lexikographischen Behandlung von polylexikalen Lexikon-Einheiten bedeutend bei; vor allem aus der Sicht der neueren Betrachtungen des Lexikons aus der Perspektive der Konstruktionsgrammatik.

Schlüsselwörter: MODUL WERBESLOGANS, ONLINE-SLOGAN-ARTIKEL, SLOGANGEBRAUCH, SLOGAN-VARIANTEN, SLOGAN-MUSTER, LEXIKOGRAFISCHE RESSOURCEN, KORPUSINFORMIERTE INTERNETLEXIKOGRAFIE, LEXIKOGRAFISCHE ONLINE-SLOGANARTIKEL

Abstract: E-Module Werbeslogans. A Corpus Informed Lexicographic Res-
source of Advertising Slogan Use Outside the Domain of Advertising. The
article aims to describe the development of E-Module Werbeslogans, integrated into OWID-Sprich-
wörterbuch at the Leibniz Institute for German Language in Mannheim. It represents a corpus 
informed online description and presentation of advertising slogans, i.e. set sentences from advertising, currently used outside the domain of advertising in everyday language. As these advertising slogans are used in the same way as proverbs, they can be similarly lexicographically described. The module is based on the lexicographic concepts developed in the EU project SprichWort. Eine Internetplattform für das Sprachenlernen für die Sprichwort-Plattform as well as the proverb articles in the OWID-Sprichwörterbuch; it is innovative and does not represent a continuation of traditional dictionaries in the field of phraseology. The article describes the corpus informed method for the analysis of advertising slogans used outside of the domain advertising, continues with the description of the lexicographic process as well as the microstructure of online slogan articles and concludes with the links that connect different information at the macrostructural level of OWID (Online-Wortschatz-Informationssystem Deutsch).

Keywords: MODULE WERBESLOGANS, ONLINE SLOGAN ARTICLES, ADVERTISING SLOGAN USE, SLOGAN VARIANTS, SLOGAN PATTERNS, LEXICOGRAPHIC RESSOURCES, CORPUS INFORMED INTERNETLEXICOGRAPHY, LEXICOGRAPHIC ONLINE-SLOGANARTICLES

\section{Einleitung}

Heute steht einem eine Vielfalt an unterschiedlichen, online frei verfügbaren lexikografischen Ressourcen in vielen Sprachen zur Verfügung, denn die lexikografische Forschung und Praxis haben sich seit Mitte der 90er Jahre durch das Internet, den Computereinsatz und die Untersuchungen umfangreicher elektronischer Korpora ${ }^{1}$ funktional und strukturell stark verändert und modifiziert (Stichwort: Internetlexikografie) (vgl. Klosa und Müller-Spitzer 2016: XI).

Wörterbuchportale, semi-automatisch generierte Angaben, komplexe Suchfunktionen und ein moderater Umfang an Multimodalität sind heute Usus. Auch die lexikographische Arbeitsumgebung spiegelt diese Entwicklung wider mit der Verbindung zwischen Korpora und intelligenten Tools zur Extraktion von Daten und verschiedenen Möglichkeiten der Verbindung $\mathrm{zu}$ texttechnologischen Anwendungen (Engelberg, Klosa-Kückelhaus und Müller-Spitzer 2019: 30).

Lexikografische Online-Ressourcen zur deutschen Sprache werden beispielsweise vom Dudenverlag (Duden Onlinewörterbuch), von der Union deutscher Akademien (z. B. DWDS, Wörterbuchnetz u. a.) und auch am Leibniz-Institut für Deutsche Sprache in Mannheim (OWID) (vgl. Klosa-Kückelhaus und MüllerSpitzer 2019: 418f.) erstellt. Im Wörterbuchportal OWID (Online-WortschatzInformationssystem Deutsch), das sich auf "Ressourcen zu spezialisierten Wortschatzbereichen konzentriert" (ebd.), stehen den User/-innen zehn lexikografische Ressourcen zur Verfügung (Stand März 2021)², darunter auch das OWID-Sprichwörterbuch, eine korpusbasierte und „nach Kriterien der wissenschaftlichen Lexikografie erarbeitete Dokumentation aktuell gebräuchlicher fester Sätze der deutschen Sprache - im Kern Sprichwörter" (OWID-Sprichwörterbuch $)^{3}$ (Abb. 1). Ins Sprichwörterbuch wurde zuletzt das Modul Werbeslogans (Steyer und Polajnar 2015) integriert. Hierbei handelt es sich um die 
lexikografische Beschreibung des aktuellen Korpusgebrauchs von Werbeslogans, die in der Gemeinsprache eine gewisse Geläufigkeit aufweisen. Da diese Slogans ähnlich wie Sprichwörter funktionieren, wurden sie nach demselben Modell (Steyer 2012; Steyer und Ďurčo 2013; Durčo, Steyer und Hein 2017) mit einigen Änderungen in der Wörterbuchartikel-Struktur beschrieben und in das Sprichwörterbuch integriert. Die Slogan-Artikel zeigen nicht nur, wie lebendig und variabel Werbeslogans in außerwerblichen Kontexten sind und wozu Sprecher/-innen sie benutzen, sondern auch, dass die Werbesprache eine moderne Quelle für die Entstehung neuer Sprichwörter darstellt.

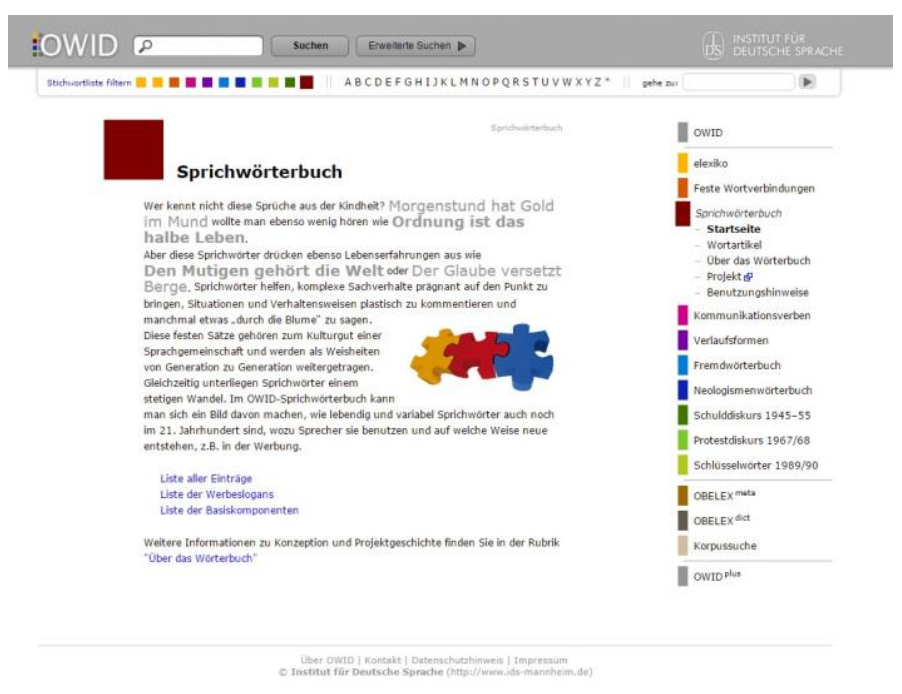

Abb. 1: Das OWID-Sprichwörterbuch mit der Liste der Werbeslogans und weiteren lexikografischen Ressourcen des Wörterbuchportals OWID (rechte Spalte)

Dem Modul Werbeslogans liegt eine umfangreiche korpusinformierte Untersuchung zugrunde. Darin wurde der Slogangebrauch in Zeitungskorpora des Deutschen Referenzkorpus (vgl. DEREKO) im Zeitraum 1990-2008 diachron untersucht und die Mikrodiachronie ihres Vorkommens sowie die Tendenzen ihres Bedeutungswandels, ihrer Varianz und Musterbildung und ihrer kontextuellen Einbettung in außerwerblichen Kontexten nachgezeichnet (Polajnar 2012, 2016, 2019).

Das neue Modul ist ähnlich wie das Sprichwörterbuch selbst eine korpusinformierte und "nach Kriterien der wissenschaftlichen Lexikografie erarbeitete Onlinedokumentation aktuell gebräuchlicher verfestigter Sätze" (OWID-Sprichwörterbuch) aus der Werbung. Die Dokumentation von Werbeslogans wird mithilfe systematischer, empirischer Erhebungen mit COSMAS II (DEREKO) und anschließend mit lexpan (2017), dem Analysewerkzeug zur Systematisierung von KWIC-Zeilen, erarbeitet. Folglich wird nicht an die Fortschreibung tra- 
dierter Wörterbücher angeknüpft. Das Modul basiert einerseits auf den lexikografischen Konzepten, die im EU-Projekt SprichWort. Eine Internetplattform für das Sprachenlernen für die Sprichwort-Plattform entwickelt wurden (vgl. SWP; Steyer 2012; Steyer und Ďurčo 2013; Ďurčo, Steyer und Hein 2017; Jesenšek 2011, 2013), und andererseits auf der im Folgenden vorgestellten korpusinformierten Untersuchung.

Der vorliegende Artikel bemüht sich um eine transparente Darstellung der korpusinformierten Methodik für die Untersuchung des Slogangebrauchs (Abschn. 3 und 4), des lexikografischen Prozesses, der Konzeption der Mikrostruktur der Slogan-Artikel (Abschn. 5) sowie die verschiedenen Zugriffsmöglichkeiten in OWID (Abschn. 6). Darüber hinaus wird ein Einblick in die bisherigen lexikografischen Versuche der Sloganbeschreibung gegeben (Abschn. 2).

\section{Werbeslogans in Nachschlagewerken}

Lexikografisch wurden Slogans bis dato zwar einerseits in klassischen oder online-zugänglichen Zitatensammlungen (Büchmann 1864, 422001; Duden 12 (32008); Jeromin 1969; Redensarten-Index; Liste geflügelter Worte) und andererseits in Sloganlexika (Hars 2002) erfasst, allerdings wurden in diesen Nachschlagewerken Slogans fast nie eigenständig und oft nicht systematisch sowie korpusbasiert beschrieben. Interessanterweise waren in der Ausgabe des Dudenbands 12 Zitate und Aussprüche aus dem Jahr 200215 Werbeslogans, in der kommenden Ausgabe aus dem Jahr 2008 bereits 31 Werbeslogans verzeichnet, was auf das Interesse bzw. Offenheit der Sprachgemeinschaft für moderne Sprüche sowie „auf die Präsenz und Tendenz zur Usualisierung von Slogans im aktuellen Sprachgebrauch hinweist" (Polajnar 2019: 45); in den Wörterbuchartikeln des Dudenbands 12 sind Werbeslogans nicht systematisch beschrieben und deren Gebrauch ist nur relativ selten mit Belegen aus elektronischen Korpora veranschlaulicht. Auch in den lexikografischen Online-Ressourcen sind nur vereinzelte Werbeslogans verzeichnet. Zudem wird in den Wörterbuchartikeln dieser Ressourcen eher auf die Entstehungsgeschichte als auf den Gebrauch im Alltag bzw. auf den Korpusgebrauch eingegangen. Auch das umfangreichste Nachschlagewerk zu Werbeslogans von Wolfgang Hars (2002), Das Lexikon der Werbesprüche. 500 bekannte deutsche Werbeslogans und ihre Geschichte, fokussiert die werbegeschichtlichen Informationen und es wird nur punktuell auf ihren Gebrauch eingegangen. Es kann also festgehalten werden, „dass bis dato korpusinformierte Untersuchungen zu Slogans im aktuellen Sprachgebrauch fehl[t]en" (Polajnar 2019: 14) und eine nach Kriterien der wissenschaftlichen Lexikografie systematisch erarbeitete Onlinedokumentation von Slogans wie die vorliegende als Desideratum galt. Dies hängt damit zusammen, dass Werbeslogans bis dato nicht korpuslinguistisch systematisch untersucht wurden, was die Grundlage für eine systematische, korpusinformierte, lexikografische Beschreibung und Dokumentation darstellt. ${ }^{4}$

Werbeslogans wurden bis dato als Gegenstand unterschiedlicher Forschungsdisziplinen behandelt, vorwiegend in der Werbesprachenforschung 
und zwar als Bausteine der Werbung im Hinblick auf ihre Form, Inhalt und Funktion. Darüber hinaus wurden Werbeslogans als eigenständige Bausteine bzw. Texte im Hinblick auf ihre Einbindung in außerwerbliche Kontexte aus Sicht der Textlinguistik (vgl. Fix 1997, 2007; Janich 1997, 2019), Jugendsprachenforschung (vgl. Androutsopoulos 1997; Schlobinski 1989), Medienlinguistik (vgl. Betz 2006) sowie Phraseologie (vgl. Burger, Buhofer und Sialm 1982; Lüger 1999) untersucht (vgl. Polajnar 2019: 19-51). Hierbei entstanden einige textlinguistische und phraseologische Definitions- und Klassifikationsversuche, die den Slogan entweder als Werbespruch (Hemmi 1994: 62), Spruchtextsorte (Fix 2007: 464), geflügeltes Wort (Janich 1997) oder satzwertigen Phraseologismus (Lüger 1999; Burger 2015) auffassten; das Prinzip ihrer Wiederverwendung bzw. Verselbstständigung wurde im Hinblick auf Intertextualität (vgl. Fix 1997; Janich 1997) oder Phraseologisierung mit den Stufen Zitat, geflügeltes Wort, satzwertiger Phraseologismus (vgl. Burger, Buhofer und Sialm 1982: 56) perspektiviert. Die einzige korpusinformierte Untersuchung zum aktuellen Slogangebrauch außerhalb Werbung ist die Untersuchung von Polajnar (2019), die dem Modul Werbeslogans zugrunde liegt.

\section{Korpusinformierter Zugang zum Slogangebrauch außerhalb der Domäne Werbung}

Im Mittelpunkt der Untersuchung, die dem Modul Werbeslogans zugrunde liegt, standen ",ausgewählte deutschsprachige Werbeslogans mit hohem Wiedererkennungswert und einer Tendenz zur Usualisierung im aktuellen Sprachgebrauch" (Polajnar 2019: 11). Ihre lexikalische Verfestigung konnte korpusinformiert $^{5}$ anhand umfangreicher elektronischer Korpora (DEREKO) validiert und rekonstruiert werden. In die Untersuchung wurden 44 kodifizierte Werbeslogans miteinbezogen, die zwei Slogansammlungen mit Referenzcharakter entstammen (Duden 122008 und eine Liste der bekanntesten (Firmen-)Slogans vom Internetportal slogans.de). „Für die Beschreibung ihrer Verwendungsspezifik als eigenständige satzwertige Wortschatzeinheiten außerhalb der Domäne Werbung wird das Modell der usuellen Wortverbindungen sowie die korpuslinguistische Methodologie von Steyer (u. a. 2013, 2018) auf die Spruchtextsorte ,Slogan' angewandt" (Polajnar 2019: 11) und in einem nächsten Schritt mit weiteren qualitativen und quantitativen Methoden (vgl. Keibel 2008; Polajnar 2012) verknüpft. Die Slogan-Muster und Slogan-Varianten wurden mit dem Analysewerkzeug zur Systematisierung von KWIC-Zeilen lexpan (2017) erarbeitet.

\subsection{Iterative Phrasensuche mit engen und weiten Suchanfragen}

Die Korpusvalidierung ausgewählter Slogans und deren Varianten sowie die Identifizierung von Slogan-Mustern erfolgt mit einer unterschiedlich elaborierten iterativen Phrasensuche im Deutschen Referenzkorpus (DEREKO). Slogans wurden zunächst mithilfe einer komplexen Suchprozedur ${ }^{7}$ via COSMAS II eigens analysiert und dann anhand der Korpusevidenzen qualitativ bewertet. 
Als Beispiel seien die enge und die weite Suchanfrage ${ }^{8}$ zum Slogan $D a$ weiß man, was man hat. dargestellt und diskutiert. Die enge Suchanfrage dient der genauen Ermittlung von Korpusbelegen für die Kernform, z. B. \$da /+w1:1 weiß /+w1:1 man /+w1:1 was /+w1:1 man /+w1:1 hat. In der Regel umfassen enge Suchanfragen die im Stichwort angeführten Wortformen des Slogans und einen geringen Wortabstand; sie orientieren sich an der Form des Stichworts. Man bekommt bei dieser Suche dann alle Belege mit genau dieser Kernform. Hierbei können die ermittelten Kernformen noch sehr eng an dem werblichen Ursprungskontext gebraucht werden, oder aber haben bereits eine allgemeinere Bedeutung entwickelt, wie die folgenden Belege aus dem Korpus zeigen.

(1) HAZ09 die Vertrautheit des guten, alten „Tatorts“. Da weiß man, was man hat, HMP12 Eiche, da weiß man was man hat. Ein echter Wert und "unkaputtbar".

T12 2013 wird wieder Merkel gewählt (da weiß man, was man hat) und wir rutschen

RHP13 Ich koche jedes Jahr Marmelade ein. Da weiß man, was man hat, und sie schmeckt

NGACB Dann mache die Updates doch alle manuell. Da weiß man, was man hat.

Bei einer weiten Suchanfrage hingegen erweitert man den Wortabstand zwischen den Formen des Stichworts und/oder bezieht das ganze Flexionsparadigma ein. Mittels einer weiten Suchanfrage können lexikalische, aber auch syntaktische Varianten sowie Musterrealisierungen und dadurch SloganMuster erarbeitet werden; letztere geben des Weiteren die Auskunft über den invarianten Kern, lexikalische Füller sowie die Einschübstellen: \$da /+w1:1 (Ewissen oder wei $\beta$ ) $/+s 0$ was $/+s 0$ Ehaben (siehe $5 \mathrm{i}$. Formvarianten und $5 \mathrm{j}$. Ersetzung von Komponenten). Um eine passende weite Suchanfrage bei einem jeden Slogan zu fixieren, muss diese durch ein iteratives Verfahren feingetunt werden. So gibt die Auswahl der Konkordanzen der genannten weiten Suchanfrage Auskunft darüber, dass im Slogan Da weiß man, was man hat. ausschließlich die beiden Indefinitpronomen man durch verschiedene Personalpronomen ersetzt werden können, was bereits Indizien für den invarianten Kern Da [wissen] X, was X [haben]. liefert.

(2) U02 „Kinder, kauft Staatspapiere, da wisst ihr, was ihr habt BRZ06 pflanze ich lieber selber Bohnen, Erbsen und Möhren an. Da weiß man doch, was man hat"“. NGLD Da weißt Du, was Du hast - und musst nichts wegwerfen.

T91 auf den Kleinstadtgeschmack abgestimmt. Da weiß der Alte, was er hat.

Aus methodischer Sicht können also bereits KWIC- und Volltext-Analysen wichtige Hinweise über den invarianten Kern bzw. die Binnenstruktur eines lexikalisch geprägten Slogan-Musters, die typischen Slogan-Varianten sowie Gebrauchsrestriktionen liefern. Besonders ertragreich scheint in solchen Fällen die iterative Suchheuristik (z. B. Ausschließen von Teilkomponenten u. a.) sowie KWIC-Analysen und KWIC-Systematisierung mit Analyseprogramm lexpan (2017).

\subsection{Exploration syntagmatischer Slogan-Muster mit lexpan}

Mit dem Analysewerkzeug zur Systematisierung von KWIC-Zeilen lexpan ${ }^{9}$ (2017) 
erfolgte die systematische Analyse von Slogan-Varianten und syntagmatischen Slogan-Mustern und zwar mithilfe von exportierten KWIC- und Kookkurrenzlisten aus DEREKO. ${ }^{10}$ Das einzelsprachenunabhängige Analyseprogramm dient „der explorativen Untersuchung von Festigkeit, Varianz, Slotbesetzungen und kontextuellen Einbettungsmustern syntagmatischer Strukturen" (ebd.).

Mithilfe von lexpan konnten die zunächst manuell erarbeiteten Ergebnisse in folgenden Bereichen automatisch verifiziert werden. Zum einen lassen sich mit dem Analysewerkzeug Slogan-Muster explorieren, indem Leerstellen entdeckt und systematisch untersuchen werden. Zum anderen kann man auf diese Weise den Kontext von Slogans bzw. ihre kontextuelle Einbettung analysieren, indem man typische kontextuelle metakommunikative Marker und minimale lexikalische Einschübe systematisch untersucht. „Der Mehrwert von lexpan gegenüber manuellen Analysen erweist sich nicht nur in einer viel einfacheren, systematischen Identifikation von einbettenden Elementen, sondern auch von kontextuellen Einbettungsmustern." (Polajnar 2019: 65)

Um mit lexpan den Ikea-Werbeslogans Wohnst du noch, oder lebst du schon? auf Musterhaftigkeit bottom up anhand lexikalischer Musterrealisierungen zu rekonstruieren, muss man mit einer weiten Suchanfrage im virtuellen Zeitungskorpus noch /+w1:1 „oder" /+w3:3 schon eine KWIC-Liste ermittelt. Nach dem Exportieren in lexpan wird die KWIC-Liste mit dem Suchmuster \# \# noch oder \# \# schon durchsucht. Die automatische Auswertung der Y-Leerstellen ergibt, dass diese auf Personalpronomen restringiert sind, darunter überproportional häufig auf das Personalpronomen $d u-d u$ bzw. $D u-D u(78,40 \%)$. Daraus ergibt sich, dass der lexikalisch geprägte Slogan-Muster $X d u$ noch, oder $Y d u$ schon? eine prototypische Teilrealisierung des abstrakteren Musters darstellt. Allerdings weisen auch weitere Personalpronomina gewisse Vorkommenshäufigkeit auf: Sie-Sie $(6,64 \%)$, ihr-ihr $(2,66 \%)$, sie-sie $(1,66 \%)$, wir-wir $(1 \%)$, er-er $(0,66 \%)$ und es-es $(0,66 \%)$ (Abb. 2$)$.

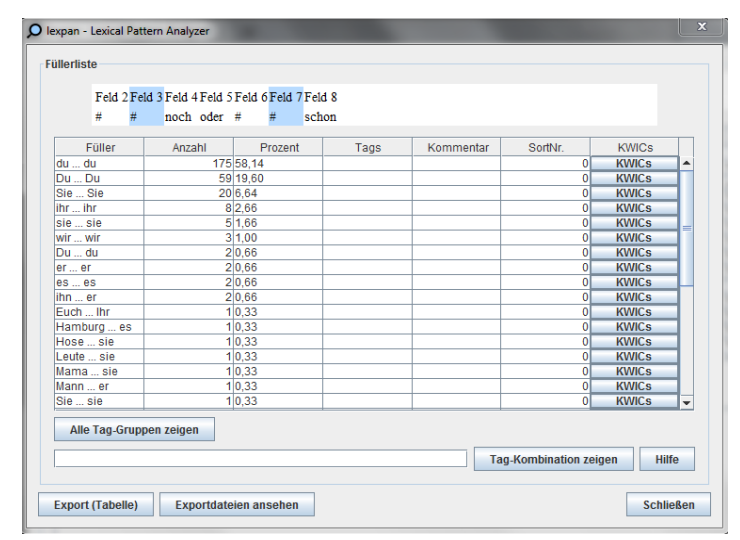

Abb. 2: Schnappschuss der automatisch erstellten Lückenfüllertabelle für (0-3 Leerstellen) noch oder (0-3 Leerstellen) schon (Quelle: lexpan: Ausschnitt) 
Analysiert man zugleich alle vier Slots des abstrakten Musters, ergibt die Füllerliste aus lexpan, dass der Slogan Wohnst du/Du noch, oder lebst $d u / D u$ schon? mit 15,28 \% als der prototypische Vertreter gilt, da diese Verb-PronomenKombination im Vergleich zu den anderen Kombinationen eine relativ hohe Vorkommenshäufigkeit aufweist (Abb. 3). Unter den häufigeren lässt sich auch die umgekehrte Kombination beider Verben wohnen und leben konstatieren, die als eine syntaktische Slogan-Variante aufzufassen ist: Lebst du/Du noch, oder wohnst $d u / D$ u schon? $(2,66 \%)$.

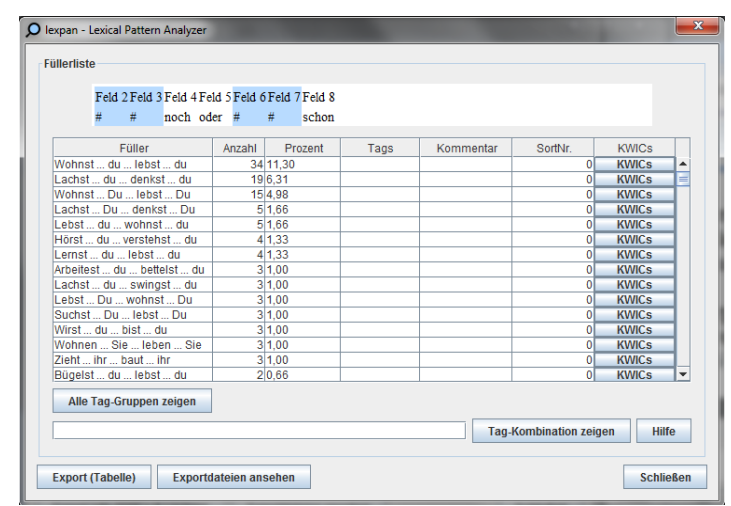

Abb. 3: Schnappschuss der automatisch erstellten Lückenfüllertabelle für (0-3 Leerstellen) (0-3 Leerstellen) noch oder (0-3 Leerstellen) (0-3 Leerstellen) schon (Quelle: lexpan: Ausschnitt)

\section{OWID-Sloganliste}

Vor der lexikografischen Beschreibung muss man sich zunächst den Selektionsmethoden bzw. der Sloganauswahl widmen. Die hiesige Stichwort-Liste der Werbeslogans stellt eine Auswahl der häufigsten Werbeslogans dar, die den Gegenstand der oben erwähnten korpusinformierten Untersuchung zum Slogangebrauch in Zeitungskorpora des DEREKO bildeten. Die ersten 30 Slogans mit höchster Vorkommenshäufigkeit wurden im nächsten Analyseschritt auf ihre "Satzwertigkeit" (,satzwertige Phraseologismen“ Lüger 1998, 1999) hin genauer untersucht, weil sich Satzwertigkeit bereits bei der Sprichwortidentifizierung als empirisch gut operationalisierbares Kriterium erwiesen hat (vgl. Steyer und Ďurčo 2013). Der nichtsatzwertige Gebrauch bzw. die Einbettung des Werbeslogans in die Satzstruktur hat nämlich oft seine Dekomposition zur Folge. Werden Werbeslogans, wenn auch im Korpus häufig vorkommend, also vorwiegend in die Satzstruktur eingebunden gebraucht, so wurden sie in das Modul nicht aufgenommen. Als Beispiel seien Belege zweier ausgesonderten Slogans Das einzig Wahre. (Warsteiner, 1972) und Der Duft der großen weiten Welt! (Peter Stuyvesant, 1959) genannt, die den nichtsatzwertigen Korpusgebrauch veranschaulichen. 
(3) NUN90 das ich liebe, das habe ich bis zur Langweile wiederholt, das einzig wahre Rimini ist das, was ich im Studio gebaut habe."

NUN90 Appell auch all jene erreicht, die glauben, unser System sei das einzig wahre_auf dieser Welt. T90 idealistischen Logik erscheint das fortschreitende Ganze als das einzig Wahre, welches von den "Achtundsechzigern" zwanzig Jahre lang

(4) NUN90 "Vielleicht fehlt ein bißchen der Duft der großen weiten Welt im Vergleich zu anderen Berufen?"

T90 Die kannten den Duft der großen weiten Welt schon und rauchten den ganzen Abend Joints T90 Die waren ja noch nie in Italien und Spanien und wollen den Duft der großen weiten Welt auch mal schnuppern.

Im Modul Werbeslogans sind nur solche satzwertigen Werbeslogans vorzufinden, die sich bereits von ihrem Ursprungskontext (Produktwerbung) entfernt haben und eine Tendenz zum "Weisheitssatz" aufweisen. Hierbei wurden in der OWID-Sloganliste (Abb. 4) Werbeslogans aus unterschiedlichen Jahrzehnten des 20. und 21. Jahrhunderts lexikografisch erfasst:

- aus den 20ern: Nie war er so wertvoll wie heute. (Klosterfrau Melissengeist, 1925),

- aus den 50ern: Mach mal Pause-Serie (Coca-Cola, 1955), Mit fünf Mark sind Sie dabei. (ARD-Fernsehlotterie, 1956), Er läuft und läuft und läuft ... (Volkswagen, 1959) und Der Duft der großen weiten Welt! (Peter Stuyvesant, 1959),

- aus den 60ern: Wer wird denn gleich an die Luft gehen? (HB, 1960), Alle reden vom Wetter, wir nicht. (Deutsche Bahn, 1966), Da weiß man, was man hat. (Volkswagen, 1969),

- aus den 70ern: Quadratisch, praktisch, gut. (Ritter Sport, 1970), Die zarteste Versuchung, seit es Schokolade gibt. (Milka, 1971), Das einzig Wahre. (Warsteiner, 1972), Es gibt viel zu tun. Packen wir's an. (Esso, 1974), Nicht immer, aber immer öfter. (Clausthaler, 1979),

- aus den 80ern: Man gönnt sich ja sonst nichts. (Malteserkreuz Aquavit, 1985), Wir machen den Weg frei. (Volks- und Reiffeisenbanken, 1988),

- aus den 90ern: Nichts ist unmöglich. (Toyota, 1992), Ich bin doch nicht blöd. (Media Markt, 1996) oder Ja is' denn heut' scho' Weihnachten. (E-Plus, 1998) und

- aus dem 21. Jh.: Geiz ist geil! (Saturn, 2001), Wohnst du noch oder lebst du schon? (Ikea, 2002).

Die empirischen Untersuchungen haben gezeigt, dass Werbeslogans oft, aber nicht immer eine auffällige sprachliche Struktur aufweisen wie Wohnst $d u$ noch oder lebst $d u$ schon? (Ikea, 2002). In einigen Fällen basieren sie allerdings auf völlig regulären, strukturell unauffälligen Sätzen (z. B. Ich liebe es (McDonald's, 2003), Mit fünf Mark sind Sie dabei. (ARD-Fernsehlotterie, 1956)), die zum Teil umgangssprachlichen oder dialektalen Charakter aufweisen (z. B. Ich bin doch nicht blöd. (Media Markt, 1996), Es gibt viel zu tun. Packen wir's an. (Esso, 1974) sowie Ja is' denn heut' scho' Weihnachten (E-Plus, 1998)). Erst durch die Verknüpfung mit einer Marke bzw. einem Produkt und durch vielfaches Wiederholen treten sie 
aus dem Fluss der Werbekommunikation heraus. Die Sprachgemeinschaft entscheidet dann schließlich darüber, ob der Slogan genug "Spruch-Potenzial" hat, d. h. Alltagssituationen, Verhaltensweisen und Normen plastisch kommentiert und auf den Punkt bringt und damit die Chance besitzt, in den Sprachbestand auf Dauer überzugehen.

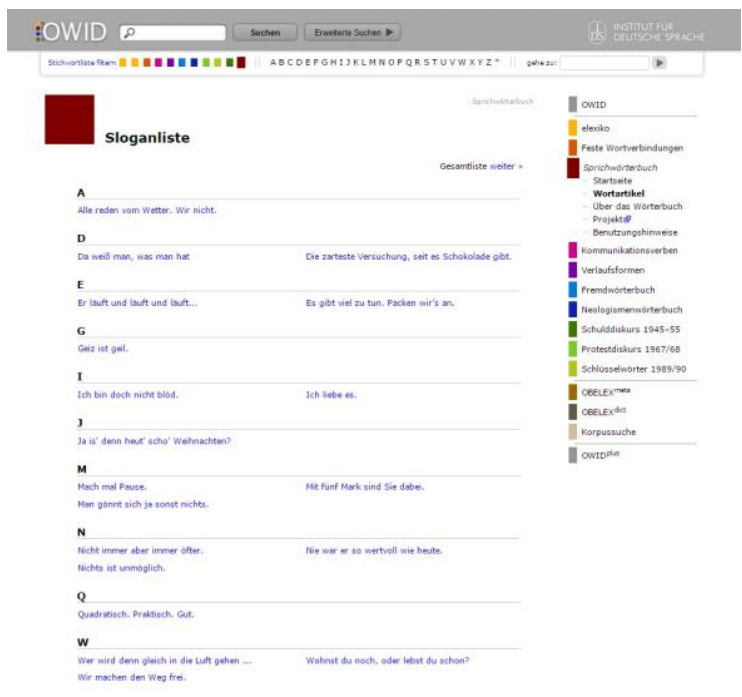

Abb. 4: OWID-Sloganliste

\section{Aufbau der Slogan-Artikel}

Die korpusbasierte Beschreibung umfasst folgende Bausteine: „Kernform”, „Basiskomponenten”, „Äquivalente in anderen Sprachen” (falls vorhanden), "Suchanfragen für Recherche im Korpus", "Geschichte”, „Bedeutung”, "Gebrauchsbesonderheiten”, „Formvarianten”, „Ersetzung von Komponenten”, "Typische Verwendung im Text" und „Vorkommen in Nachschlagewerken”. Die Slogan-Artikel unterscheiden sich von den anderen SprichwörterbuchArtikeln durch folgende Strukturmerkmale: In den Bausteinen „Kernform”, "Äquivalente" und "Geschichte" (siehe dazu 5a, 5c und 5e) wird auf die Entstehungsgeschichte als (internationaler) Firmen-, Marken- oder Produktslogan verwiesen; zudem wird am Ende der Slogan-Artikel im Baustein „Vorkommen in Nachschlagewerken" (5k) ihre bisherige lexikografische Erfassung evidentiert. Im Folgenden sollen die einzelnen Bausteine der Slogan-Artikel anhand von Beispielen dargestellt und kommentiert werden.

\section{a. Kernform}

Obwohl man bei Werbeslogans intuitiv in die Versuchung geraten könnte, den 
Originalslogan als Kernform anzusetzen, zeigt die empirisch fundierte und hiermit methodisch sichere Korpusanalyse (Abschn. 3) ein anderes Bild. Die Kernform stellt folglich die im Korpus häufigste satzwertige Form des Werbeslogans dar; diese ist im Vergleich zum Originalslogan oft ohne Angabe des Markennamens. Die Korpusanalyse, mit welcher die auffällig rekurrente Vorkommensform identifiziert wird, hat gezeigt, dass Markennamen oft eine Hürde bei der Verselbstständigung von Slogans darstellen. Dies ist auf ihre Identifikationsfunktion des Unternehmens bzw. der Marke und hiermit mit dem Verweis auf die Ursprungsdomäne zurückzuführen. So können Werbeslogans, bei denen der Markenname Teil der Satzstruktur ist, erst auf der Musterebene eine usuelle, situationsunabhängig-abstrakte (nicht-werbliche) Bedeutung erlangen, wie dies beim Haribo-Slogan X macht Kinder froh. deutlich wird.

Bei den meisten Werbeslogans entspricht die Kernform dem Slogan ohne den Markennamen. Zudem liegen Slogan-Artikel mit Kernformen vor, die eine verkürzte Form des Originalslogans darstellen: Z. B. wurde die Kernform vom Werbeslogan für die Deutsche Bahn aus dem Jahr 1966 Alle reden vom Wetter. Wir nicht. Fahr lieber mit der Bundesbahn. anhand von Korpusvorkommen auf Alle reden vom Wetter. Wir nicht. angesetzt. Unter der Kernform wird im Werbeslogan-Artikel deshalb immer auch der Originalslogan mit dem Markennamen aufgeführt (Abb. 5).

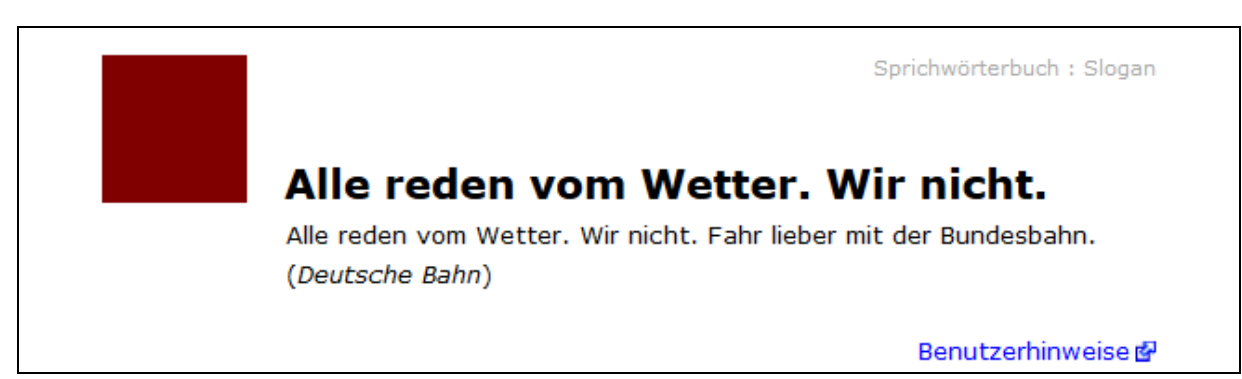

Abb. 5: OWID-Sloganartikel: Stichwort Alle reden vom Wetter. Wir nicht.

Wie die Sprichwörter weisen auch die meisten Werbeslogans eine eindeutige Kernform auf. Bei einigen Werbeslogans liegen jedoch mehrere Varianten vor, bei denen sich eine als prototypisch erweist (Kernform) und die anderen unter Formvarianten aufgeführt werden (siehe dazu auch h) (vgl. Steyer und Ďurčo 2013).

Kernform: Es gibt viel zu tun. Packen wir's an.

Formvarianten: Es gibt viel zu tun. Packen wir es an.; Es gibt viel zu tun. Packt es an.; Packen wir es an.; Packen wir's an.; Packen Sie es an.

Bei Werbeslogans, die beispielsweise verselbstständigte Sätze aus Werbespots 
darstellen (gebundene Slogans) und nur gesprochen realisiert werden, konkurrieren mehrere (orthografische) Varianten miteinander, von denen keine als prototypisch gelten kann. In einem solchen Fall muss eine kompetenzbasierte Entscheidung getroffen werden. Als Beispiel sei der Werbeslogan für E-Plus genannt:

Kernform: Ja is' denn heut' scho' Weihnachten?

Formvarianten: Ja is' denn heut' schon Weihnachten?; Ja ist denn heut' scho' Weihnachten?; Ja ist denn heute schon Weihnachten?

\section{b. Basiskomponenten}

Alle lexikalischen Komponenten des Slogans (außer bestimmtem und unbestimmtem Artikel) werden zwecks Verlinkung mit dem elexiko-Wörterbuch in OWID und der Ermöglichung unterschiedlicher Zugriffsmöglichkeiten gesondert ausgezeichnet.

\section{c. Äquivalente in anderen Sprachen}

Diese Rubrik wird in all jenen Fällen bearbeitet, in denen Webseiten unterschiedlichen Charakters mit Äquivalenten in anderen Sprachen gefunden wurden (Wikipedia, Firmenseiten usw.): In der Regel handelt es sich um Werbeslogans international agierender Unternehmen, wie z. B. Milka: Die zarteste Versuchung, seit es Schokolade gibt., McDonald's: Ich liebe es. oder Media Markt: Ich bin doch nicht blöd., die wegen europaweiten oder weltweiten Präsenz Marketing bedingte Äquivalente in unterschiedlichen Sprachen aufweisen. Bei den Äquivalenten internationaler Slogans handelt es sich meist um Teiläquivalente, vereinzelt wurden auch semantische Äquivalente und Nulläquivalente konstatiert (Polajnar 2016). Ob diese in den jeweiligen Sprachen genauso wie der Originalslogan in außerwerblichen Kontexten Verwendung finden, müsste einzeln für jede Äquivalente wie im Deutschen korpusbasiert überprüft werden. Eine exemplarische Untersuchung von Sloganäquivalenten (Polajnar 2016) hat jedoch zeigen können, dass viele populäre internationale Slogans nicht nur in der Ausgangssprache bzw. im Deutschen, sondern auch in den anderen Sprachen erneut in außerwerblichen Kontexten Verwendung finden und sogar Variationsmuster bilden (z. B. für Snickers im Slowenischen: $X$ si ful drugačen. (' $X$ bist du ganz anders') sowie für Red Bull im Englischen: .X gives you wings. usw.).

\section{d. Suchanfrage für Recherche im Korpus}

Diese bereits im großen OWID-Sprichwörterbuch integrierte Angabe soll der schnellen Auffindbarkeit von authentischen Sprachbelegen im Deutschen Referenzkorpus $(D E R E K O)$ dienen. Die Suche nach komplexen Sätzen stellt gerade für einen unerfahrenen Nutzer oft eine erhebliche Hürde dar, muss er sich doch einer manchmal recht komplizierten Syntax bedienen. Die hinterlegten 
Suchanfragen können nun ganz problemlos in das COSMAS II-Suchfenster kopiert werden. Ein neues OWID-Feature wird demnächst diese Suche noch weiter erleichtern. Der Nutzer muss dann nur noch auf die Suchanfrage klicken, und die Korpusanalyse startet automatisch im Hintergrund. Der Nutzer kann so alle Originaltextstellen, in denen der Slogan vorkommt, immer auf dem jeweils aktuellen Stand von DEREKO erhalten. Es werden eine enge und eine weite Suchanfrage für weitere eigenständige Recherchen im Korpus aufgeführt (vgl. Abschn. 3.1).

\section{e. Geschichte}

Anders als bei den Sprichwörtern war es bei den Slogans wichtig, auf ihren ursprünglichen Gebrauch in der Werbung bzw. ihre Entstehungsgeschichte sowie auf ihre domänenspezifische Bedeutung zu verweisen. Weil der Ursprung bzw. die Quelle von Werbeslogans nachgewiesen werden kann, werden Slogans häufig als zeitspezifische geflügelte Worte der jüngsten Vergangenheit bezeichnet (vgl. Janich 2010: 61). Doch die Slogan-Artikel zeigen, dass Slogans nicht nur zitiert werden, sondern Varianten und vor allem Muster mit Leerstellen aufweisen, die einem ermöglichen, sie durch das Aufgreifen von Schlüsselwörtern an beliebige Kontexte anzupassen. Dies verweist eher auf ihren ausgeprägten musterhaften Gebrauch (vgl. Bubenhofer 2009).

In diesem Teil des Slogan-Artikels werden also Informationen zur Entstehung des Werbeslogans aus Sekundärquellen gegeben (Nachschlagewerke, Webportale und einschlägige Webseiten usw.). Angereichert werden diese Texte durch DEREKO-Belege, in denen die Entstehung oder der Ursprungskontext des jeweiligen Slogans explizit thematisiert wird und Werbeslogans in Zitatform gebraucht werden. Dadurch unterscheidet sich auch der Korpusgebrauch von Slogans in diesen Belegen vom Korpusgebrauch in allen weiteren Belegen, da hier der Werbeslogan noch keine usuelle, situationsunabhängigabstrakte (nicht-werbliche) Kernbedeutung aufweist, wie sie in weiteren Teilen der Slogan-Artikel genau beschrieben wird (siehe f). Zudem liegen hier Links auf Youtube-Videos mit den Originalwerbespots (wenn vorhanden) vor. Beim Werbeslogan der Firma Ritter Sport Quadratisch, praktisch, gut. werden in dieser Rubrik eine Vorgeschichte samt Werbespot und ein Beleg, der auf die Werbung rekurriert (Abb. 6), dargestellt:

\section{Geschichte}

Die Firma Ritter Sport wirbt noch heute mit diesem Werbeslogan für die Schokolade der Marke Ritter Sport, die für ihre quadratische Form bekannt ist. "Es gibt die Schokolade in dieser Form [...] bereits seit 1932, der populäre Werbespruch wurde aber erst im Jahr 1970 entwickelt. Heute zitiert man ihn im Zusammenhang mit den verschiedensten Produkten oder Ideen meist dann, wenn man deren praktische Seite lobend hervorheben möchte" (Duden 12 2008: 434). 
Korpusbeleg

$\mathrm{Zu}$ bundesweiter Bekanntheit brachte es Ritter Sport ab 1970 mit der ersten Joghurtschokolade und den drei Worten: quadratisch, praktisch, gut. Jede Sorte erhielt ihre eigene Farbe und eine Verpackung, die sich durch einfaches Knicken öffnen lässt. (Die Rheinpfalz, 22.02.2012, S. 7; Eine süße Idee wird 100 Jahre alt)

Abb. 6: OWID-Sloganartikel: Geschichte Quadratisch, praktisch, gut. mit dem Link auf den Werbespot

\section{f. Bedeutung}

Die Bedeutungserklärungen basieren durchweg auf den authentischen Korpusvorkommen. Hier geht es um die usuelle, situationsunabhängig-abstrakte (nicht-werbliche) Kernbedeutung, die in allen Korpusverwendungen nachweisbar sein muss. Unter "Bedeutung” wird der verallgemeinerte semantische Kern erfasst, der für alle üblichen Vorkommen dieses Slogans gleichermaßen zutrifft (vgl. SWB). Hierbei verwenden wir eine weitgehend standardisierte Beschreibungssprache, die bereits im EU-Projekt Sprichwort (vgl. Steyer 2012 und SprichWort-Projekt) aus fremdsprachendidaktischen Gründen eingeführt wurde, beispielsweise einleitende Formulierungen wie "Sagt man dafür, dass ..."; "Sagt man, wenn ...". Die Bedeutungsbeschreibung soll anhand zweier Beispiele illustriert werden. Für den Slogan Da weiß man, was man hat. lautet die Bedeutung wie folgt: „Sagt man, wenn man lieber auf Bewährtes setzt, als neue, aber möglicherweise riskantere Dinge anzustreben." (SWB-SL) Dazu werden Belege aus dem Korpus aufgeführt.

(5) Wie Gertrud und Reiner Baum machen es viele der inzwischen mehr als 20000 deutschen Hausbesitzer an Floridas Golfküste: Außerhalb des eigenen Urlaubs vermieten sie ihre FerienImmobilie wochenweise - am liebsten an Landsleute: "Da weiß man, was man hat!" (RheinZeitung, 28.02.1997; florida)

Der Slogan Quadratisch, praktisch, gut wird folglich paraphrasiert: „,Sagt man, wenn etwas ohne großen Aufwand seine Funktion erfüllt und Qualität hat." (SWB-SL)

(6) Auf- und Umbau funktionieren nach dem Baukastenprinzip: Die dünnen Holzplatten werden mithilfe einer neuartigen Kunststofffolie zum Würfel verspannt - fertig ist der Beistell- oder Nachttisch. Merkmal: quadratisch, praktisch, gut, ganz ohne Schrauben, Bohren oder Hämmern. (Hannoversche Allgemeine, 29.05.2010; Mobil mit Modulen)

Bedeutungsbeschreibung fällt dann anspruchsvoller aus, wenn ein Slogan überwiegend in den Kontexten vorkommt, die noch stark mit der Domäne Werbung verknüpft sind. In einigen Fällen konstituiert sich die abstrakte Bedeutung erst auf der Musterebene. So ist die Verwendung des DeutscheBahn-Slogans Alle reden vom Wetter. Wir nicht. stark an den Wetter-Kontext gebunden. Erst durch die Ersetzung des Lexems Wetter durch andere Nomina 
wie Rezession oder Benzinpreise lässt sich eine generelle Bedeutung festschreiben: „Sagt man dafür, dass man anders handelt als die Mehrheit und damit vom Gängigen abweicht." (SWB-SL)

(7) Heulen und Zähneklappern wie in vielen anderen Wirtschaftsbereichen ist in der Spielwarenbranche derzeit nicht angesagt: "Alle reden von Rezession — wir nicht", betont DSVI-Geschäftsführerin Printzen mit Verweis auf insgesamt leichte Umsatzzuwächse. "Allen Unkenrufen zum Trotz konnte sich der Spielwarenmarkt in der ersten Jahreshälfte gut behaupten", unterstreicht auch Marktforscherin Gabriele Eberl. (Nürnberger Nachrichten, 26.10.2001, S. 11; Spielwarenbranche hofft auf Umsatzplus im Gesamtjahr — Warten auf Weihnachten)

Ein vergleichbares Phänomen lässt sich beim Ikea-Slogan Wohnst du noch oder lebst du schon? feststellen. Der Slogan wird noch recht häufig in Verbindung mit dem Wohnen und der Philosophie des Möbelkonzerns Ikea gebraucht. Auch hier ist die abstraktere Bedeutung erst durch andere Verbfüller gegeben: Frierst $d u$ noch oder heizt $d u$ schon? Isst $d u$ noch oder genießt $d u$ schon? Die Bedeutung kann dan wie folgt festgeschrieben werden: „Sagt man dafür, dass zu fragen ist, ob ein Zustand noch erstrebenswert oder ein anderer zu bevorzugen ist." (SWB-SL)

(8) Nein, noch geht es nicht um die Weihnachtsgeschenke - auch wenn im Handel längst wieder Lebkuchen und Spekulatius Einzug gehalten haben und familienintern mitunter schon diskutiert wird, um wessen Weihnachtsbaum man sich an Heiligabend versammeln wird. Vorerst wird allenthalben die Frage diskutiert: „Frierst du noch oder heizt du schon?!“ Angesichts der Wetterkapriolen, die sich vom Frühling über den Sommer nahtlos in den Herbst gerettet haben, hat man in den letzten Wochen kleidungstechnisch dem Zwiebellook gefrönt: morgens Wollpullover, mittags T-Shirt, abends Daunenjacke. Aber jetzt schon heizen? (Mannheimer Morgen, 01.10.2010, S. 17)

\section{g. Gebrauchsbesonderheiten}

Unabhängig von der Grundbedeutung liegen bei Slogans zudem Gebrauchsbesonderheiten vor; diese repräsentieren das Typische in den Korpusbelegen und können nur in bestimmten konkreten Gebrauchssituationen auftreten. Unter "Gebrauchsbesonderheiten" werden konnotative, pragmatische und domänenspezifische Aspekte des Slogangebrauchs berücksichtigt, die in den Belegen oft zu beobachten sind, aber nicht für alle Vorkommen verallgemeinerbar sind: Die Beschreibungen der Gebrauchsbesonderheiten werden durch folgende Formulierungen eingeleitet: „(In den Korpusbelegen wird) häufig ...”, "typischerweise” oder „in bestimmten Korpusbelegen” (SWB). Für den Slogan Da weiß man, was man hat. lautet eine der Gebrauchsbesonderheiten wie folgt: „In den Korpusbelegen wird mit dem Slogan häufig thematisiert, dass man landwirtschaftliche Produkte aus der eigenen Heimat oder aus vertrauter Herkunft bevorzugt." (SWB-SL) 
(9) Es war 1989. Die Öffentlichkeit erfuhr erstmals vom Thema BSE, dem Rinderwahnsinn. Da konnte die schwangere Dorothe Lengert, obwohl keine Vegetarierin, "kein Fleisch mehr sehen", zumal auch eine Krankheit bei Schweinen grassierte. Die in Hochscheid geborene Försterstochter und ihr Mann Thomas, ein aus Hinzerath stammender Betriebsschlosser, suchten nach Alternativen. Denn Fleisch sollte auch weiterhin auf den Tisch. Da blieb nur die Haltung eigenen Viehs: "Da weiß man, was man hat!" (Rhein-Zeitung, 15.02.1997; Altes Glanvieh kommt — durch BSE neu in Mode)

Der Slogan Quadratisch, praktisch, gut. weist ein ganzes Spektrum von Gebrauchsbesonderheiten auf (Abb. 7):

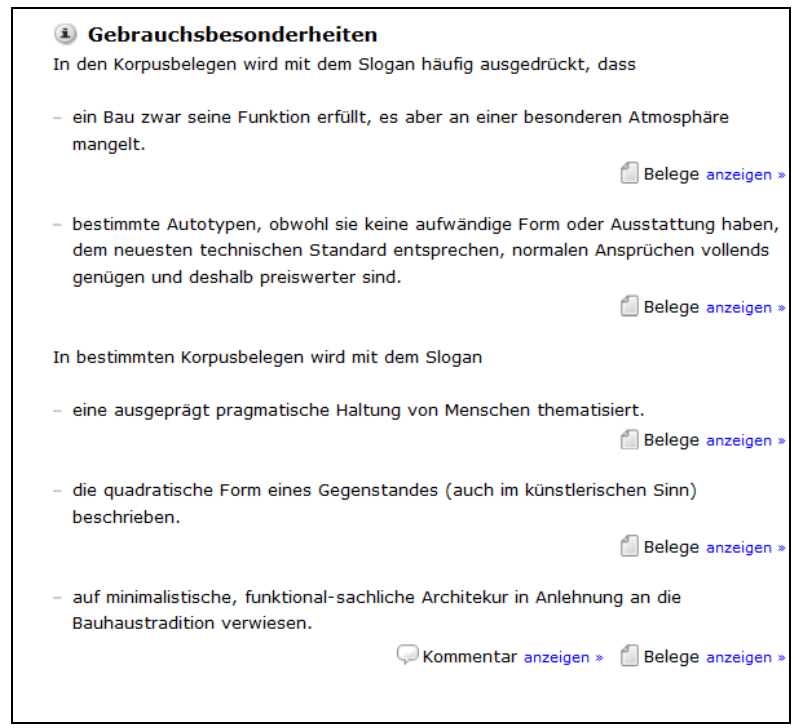

Abb. 7: OWID-Sloganartikel: Gebrauchsbesonderheiten zu Quadratisch, praktisch, gut

\section{h. Typische Formvarianten}

Formvarianten (h) gehören zusammen mit Ersetzungen von Komponenten (i) zur Varianz, die auf Usualität basiert. Unter Formvarianten sind Informationen zu allen usuellen Typen von formalen Systemvarianten einzelner SloganKomponenten oder des ganzen Slogans subsumiert (z. B. morphologische oder grammatische Varianten, orthographische Varianten). Es sind nur rekurrete Varianten verzeichnet, die bei relativ wenigen Slogans vorzufinden sind (vgl. $S W B$ ). Als Beispiel sind die morphologischen Formvarianten zum Coca-ColaSlogan Mach mal Pause. sowie die lexikalischen Formvarianten zum Slogan Mit fünf Mark sind Sie dabei. zu nennen:

Slogan-Kernform: Mach mal Pause.

Morphologische Formvarianten: Macht mal Pause. und Machen Sie mal Pause. 
Slogan-Kernform: Mit fünf Mark sind Sie dabei.

Lexikalische Formvarianten: Mit fünf Euro sind Sie dabei.

(10) Machen Sie aus dem Möbeleinkauf ein kleines Erlebnis. Gehen Sie entspannt durch die einzelnen Abteilungen oder flanieren Sie von einem Einrichtungsgeschäft zum nächsten. Setzen Sie sich nicht selbst unter Druck. Machen Sie mal Pause und gehen zwischendurch etwas essen. (Mannheimer Morgen, 22.10.1995; Wer nicht handelt, ist selber schuld)

Der Entstehung nach können Slogan-Varianten bei Werbeslogans unabhängig vom Variantentyp in zwei Gruppen unterteilt werden:

(a) domänenspezifische Slogan-Varianten, die im Rahmen der Werbekampagne(n) entstehen:

- Zu Mit 5 Mark sind Sie dabei. enstand wegen Währungsänderung eine lexikalische Variante Mit 5 Euro sind Sie dabei. (Fernsehlotterie)

- $\mathrm{Zu}$ Haribo macht Kinder froh. entstand wegen Zielgruppenerweiterung eine syntaktische Variante Haribo macht Kinder froh und Erwachsene ebenso. (Haribo)

(b) Slogan-Varianten, die im DEREKO identifiziert werden:

- Zum gesprochensprachlich markierten Slogan von E-Plus Ja, is' denn heut' scho' Weihnachten? eine orthografische Variante Ja, ist denn heute schon Weihnachten?

- $\mathrm{Zu}$ Nicht immer, aber immer öfter. eine lexikalische Variante Nicht immer, aber immer öfters.

- $\mathrm{Zu}$ Wer wird denn gleich in die Luft gehen? syntaktisch-lexikalische Varianten Aber halt — Wer wird denn gleich ... sowie Warum denn nicht gleich in die Luft gehen?

\section{i. Typische Ersetzung von Komponenten}

Hier sind Variationsmuster verzeichnet, bei denen eine Leerstelle unterschiedlich besetzt wird. Die Ersetzung von Komponenten wird durch die Korpusanalyse ermittelt; hierbei muss das Kriterium der Verallgemeinerbarkeit erfüllt werden (vgl. Steyer 2013). Die Muster-Angaben enthalten feste lexikalische Komponenten (fester lexikalischer Kern) und so genannte Füllerangaben. Im Folgenden sind Beispiele dafür aufgeführt, wie die varianten Stellen im Korpus gefüllt werden (Abb. 8). 


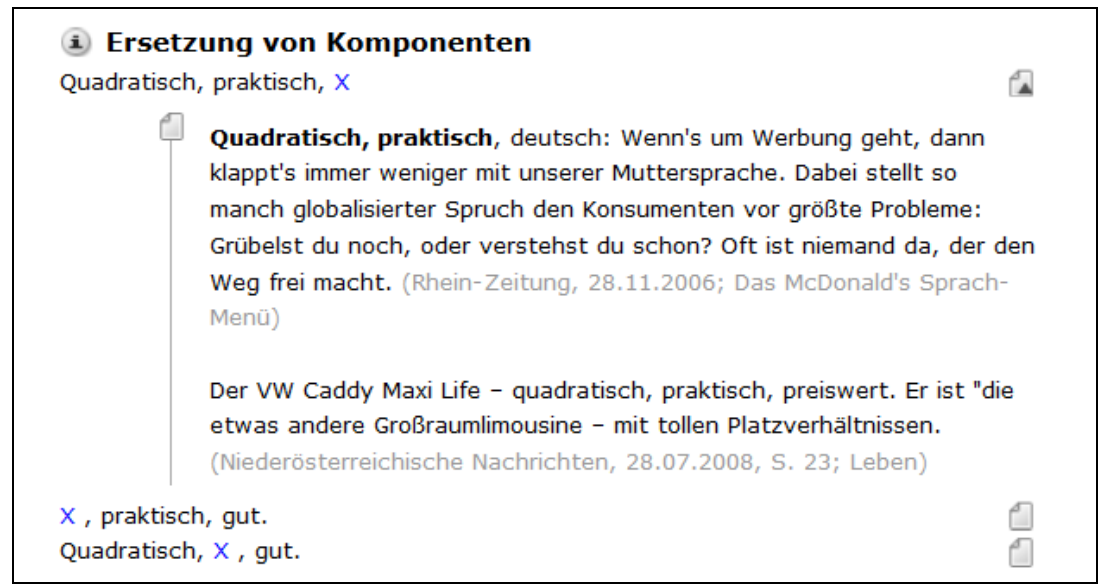

Abb. 8: OWID-Sloganartikel, Ersetzung von Komponenten zu Quadratisch, praktisch, gut

Die lexikalischen Ersetzungen können auf unterschiedlichen Ebenen und mit unterschiedlichen Effekten erfolgen, z. B.:

(a) Ersetzungen unter Beibehaltung der Sloganbedeutung

- durch formale Ersetzungen, z. B. Da weiß man, was man hat. $\rightarrow$ Da weiß ich/sie/Frau, was ich/sie/Frau habe/hat.

- durch andere Lexeme, z. B. Er läuft und läuft und läuft -> (VW-Käfer) Auto/AC Milano) läuft und läuft und läuft.

(b) Ersetzungen, die unter Beibehaltung der Sloganbedeutung auf andere Sachverhalte referieren, $\mathrm{z}$. B.

- Die zarteste Versuchung, seit es Schokolade gibt -> Die zarteste Versuchung seit es Schinken/Männer/Parteien gibt;

(c) Ersetzungen, die die Sloganbedeutung verändern, z. B.:

- Alle reden vom Wetter, wir nicht $\rightarrow$ Alle reden von/vom Zukunft/Globalisierung/Superwahljahr, wir auch; Alle reden von (der) Krise/Fußball, wir nicht.

- Es gibt viel zu tun. Packen wir es an -> Es gibt viel zu tun, lassen wir es sein/warten wir es ab/fangt schon mal an.

- Ja, is' denn heut schon Weihnachten -> Ja, is' denn heute schon Rosenmomtag/1. April/Wahlkampf (Funktionswandel: Ausdruck von unverhoffter Freude -> Ausdruck von Verwunderung)

\section{j. $\quad$ Typische Einbettungen in den Text}

Die Markierung von Slogans im neuen Kontext erfolgt durch Marker, Anführungszeichen, oft aber ohne jegliche Kennzeichnung. Diese Angabe erfasst 
jedoch ausschließlich auffällige sprachliche Phänomene in der unmittelbaren sprachlichen Umgebung eines Slogans. Solche Einbettungen steuern häufig die Interpretation auf maßgebliche Weise. Beispiele für typische Einbettungen sind "fiktive Antworten" wie die folgende Negation:

(11) Neuschnee in der Lüneburger Heide! Ja, is denn heut scho Weihnachten? Nö, aber die erste Skihalle Norddeutschlands eröffnete gestern in Bispingen. (Hamburger Morgenpost, 21.10.2006, S. 17; Ein Auftakt zum Ausschütten)

Zahlreiche Slogans werden im neuen Kotext nicht nur durch domänen- bzw. werbespezifische Termini (Werbeslogan, Werbekampagne, Werbespruch etc.) eingebettet, sondern ähnlich wie Sprichwörter durch Marker wie unter dem Motto, Devise usw. Steyer kommt anhand der korpusbasierten Untersuchung von 2000 Sprichwörtern zum Schluss, dass es in der Sprachgemeinschaft "durchaus ein ausgeprägtes Sprecherbewusstsein von ,Sätzen' gibt (nicht im grammatischen Sinne, sondern im Sinne einer funktional vollständigen Einheit)“ (Steyer 2013: 348). Allerdings scheint die Klassifizierung dieser als Sprichwort, Motto, Devise usw. für die Sprecher/-innen unwichtig. In den Sloganartikeln werden die werbespezifischen oder allgemeinen Marker nur dann vermerkt, wenn diese selbst ein Muster bilden, wie beispielsweise die Vergleiche mit einem Substantiv X à la oder X wie bei Slogan Geiz ist geil.

(12) Und auch die anderen Fraktionen im Rathaus haben sich bei der Wahl des neuen Bürgermeisters am 1. Oktober unter dem Motto "Da weiß man, was man hat" mit großer Mehrheit für den, wie damals zu hören war, "sachkundigen, fairen und zu Kompromissen bereiten Kommunalpolitiker" entschieden. (Frankfurter Allgemeine, 04.11.1997; Der neue Bürgermeister ist jetzt "an Bord")

(13) Die »MarktHallen« am Hauptgüterbahnhof stemmen sich gegen Schnell-und-Billig-Trends à la „Geiz ist geil«: Die Geschäftsleute wollen mit hochwertiger Qualität, die natürlich ihren Preis hat, und ihrem Hintergrundwissen die Kundschaft überzeugen. »Wir drücken niemandem etwas auf, sondern wir geben Erklärungen zu unseren ausschließlich biologischen Produkten«, sagt »MarktHallen«-Betreiber und Käseverkäufer Jürgen Würth. (Nürnberger Nachrichten, 15.12.2003; Einkauf mit Genuss - "MarktHallen" wollen Dornröschenschlaf beenden)

Als sprachlich auffällig werden unter typischen Einbettungen bei Slogans zudem markenspezifische Marker wie Ikea-Parole, Lotterie-Weisheit, ToyotaPrinzip genannt, also Bildungen mit Bindestrich-Komposita.

Mit fünf Mark ist man bei der Verbandsgemeinde dabei

Man muss nicht immer mit der Zeit gehen — das meint offensichtlich die VerbandsgemeindeVerwaltung Kirn-Land und hält es mit der alten Lotterie-Weisheit "Mit fünf Mark sind Sie dabei". Das sagt jedenfalls die Aufschrift an der Schrankenanlage vor dem Verwaltungsgebäude an der Bahnhofstraße aus. Doch keine Angst: Der Schlagbaum öffnet sich in diesen Euro-Zeiten auch für Nicht-Mark-Besitzer. Und wenn es keinen stört, muss man in Zeiten leerer kommunaler Kassen schließlich auch eine müde Mark für ein neues Schild ausgeben. (Rhein-Zeitung, 17.11.2004; Mit fünf Mark ist man bei der...) 


\section{k. Vorkommen in Nachschlagewerken}

Diese Angabe dient dokumentarischen Zwecken, indem vermerkt wird, ob ein Slogan bereits in irgendeiner Form kodifiziert ist.

\section{Vorkommen in Nachschlagewerken}

- Duden 12: Zitate und Aussprüche. 2008. Mannheim, Leipzig, Wien, Zürich.

- Hars, Wolfgang (2002): Nichts ist unmöglich! Lexikon der Werbesprüche. 500 bekannteste Werbeslogans und ihre Geschichte. München, Zürich.

\section{Abb. 9: OWID-Sloganartikel: Vorkommen in Nachschlagewerken}

\section{Suche und Vernetzung innerhalb des OWID-Systems}

Die Sloganartikel sind auf unterschiedliche Weise mit anderen Inhalten in OWID verbunden:

Bestimmte Slogans werden direkt mit jenen Sprichwortartikeln (SWB) verlinkt, mit denen sie in einem wie auch immer gearteten Verwendungszusammenhang stehen (vgl. Abb. 10):

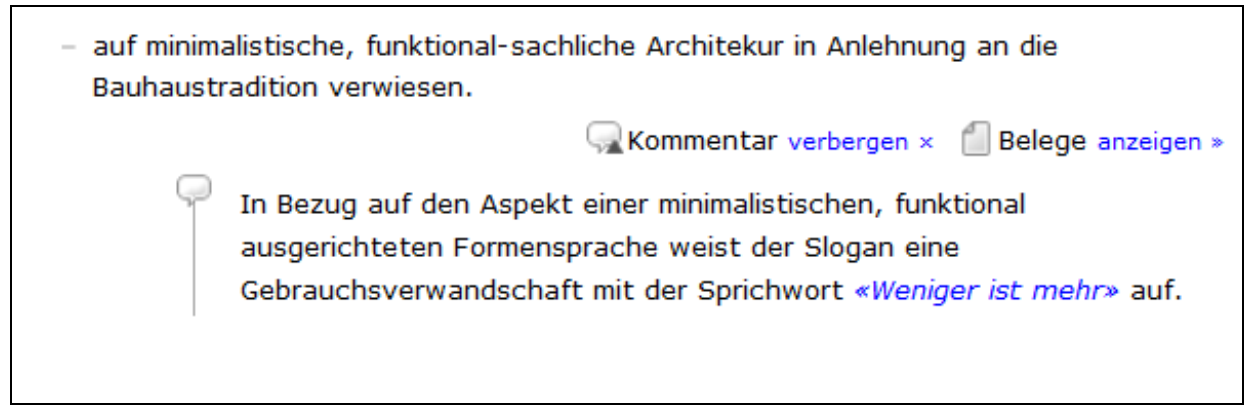

Abb. 10: Screenshot des OWID-Sloganartikels Quadratisch, praktisch, gut. -> Link zu Weniger ist mehr

Andere Beispiele für Verbindungen zu Sprichwörtern aus dem OWID-Sprichwörterbuch $(S W B)$ sind folgende:

\section{Nichts ist unmöglich:}

Wer wagt, gewinnt; Der Glaube versetzt Berge; Den Mutigen gehört die Welt; Sag niemals nie, Beharrlichkeit führt zum Ziel, Frisch gewagt ist halb gewonnen 
Im Bedeutungsaspekt des „Erst-Mal-Abwartens"

Man sollte sich nicht zu freuen; Noch ist nicht aller Tage Abend; Man soll das Fell des Bären nicht erlegen, bevor er erlegt ist; Eine Schwalbe macht noch keinen Sommer

\section{Nicht immer, aber immer öfter}

Unverhofft kommt oft; Was lange währt, wird endlich gut; Steter Tropfen höhlt den Stein; Geduld bringt Rosen

\section{Wer wird denn gleich in die Luft gehen ...}

In der Ruhe liegt die Kraft; Eile mit Weile

Durch die Auszeichnung der Basiskomponenten wird zum einen eine Verlinkung zu dem entsprechenden Einwort-Artikel in elexiko hergestellt, zum anderen kann die OWID-Suche auf die Basiskomponenten zurückgreifen. So erhält man beispielsweise bei der Komponente gut folgende Suchergebnisse (Abb. 11).

\begin{tabular}{|c|c|}
\hline 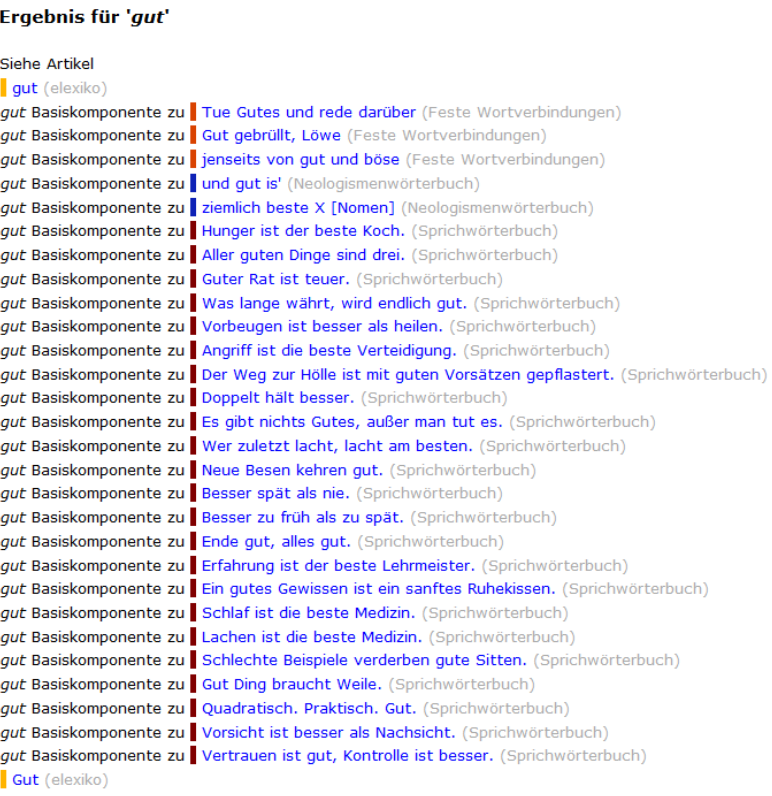 & $\begin{array}{l}\text { OWID } \\
\text { - Start } \\
\text { - Über OWID } \\
\text { - OWID zitieren } \\
\text { - Aktuelles } \\
\text { - Kontakt } \\
\text { - Datenschutzhinweis } \\
\text { - Impressum } \\
\text { elexiko } \\
\text { Paronymwörterbuch } \\
\text { Sprichwörterbuch } \\
\text { Kommunikationsverben } \\
\text { Verlaufsformen } \\
\text { Fremdwörterbuch } \\
\text { Neologismenwörterbuch } \\
\text { Schulddiskurs 1945-55 } \\
\text { Protestdiskurs 1967/68 } \\
\text { Schlüsselwörter 1989/90 } \\
\text { OBELEXmeta } \\
\text { OBELEX dict } \\
\text { Korpussuche } \\
\text { OWID plus }\end{array}$ \\
\hline
\end{tabular}

Abb. 11: Suche nach gut in OWID http://www.owid.de/suche/wort?wort=gut

Des Weiteren ist es möglich, alle Slogans und Sprichwörter nach einer Basiskomponente zusammenstellen zu lassen (Abb. 12): 


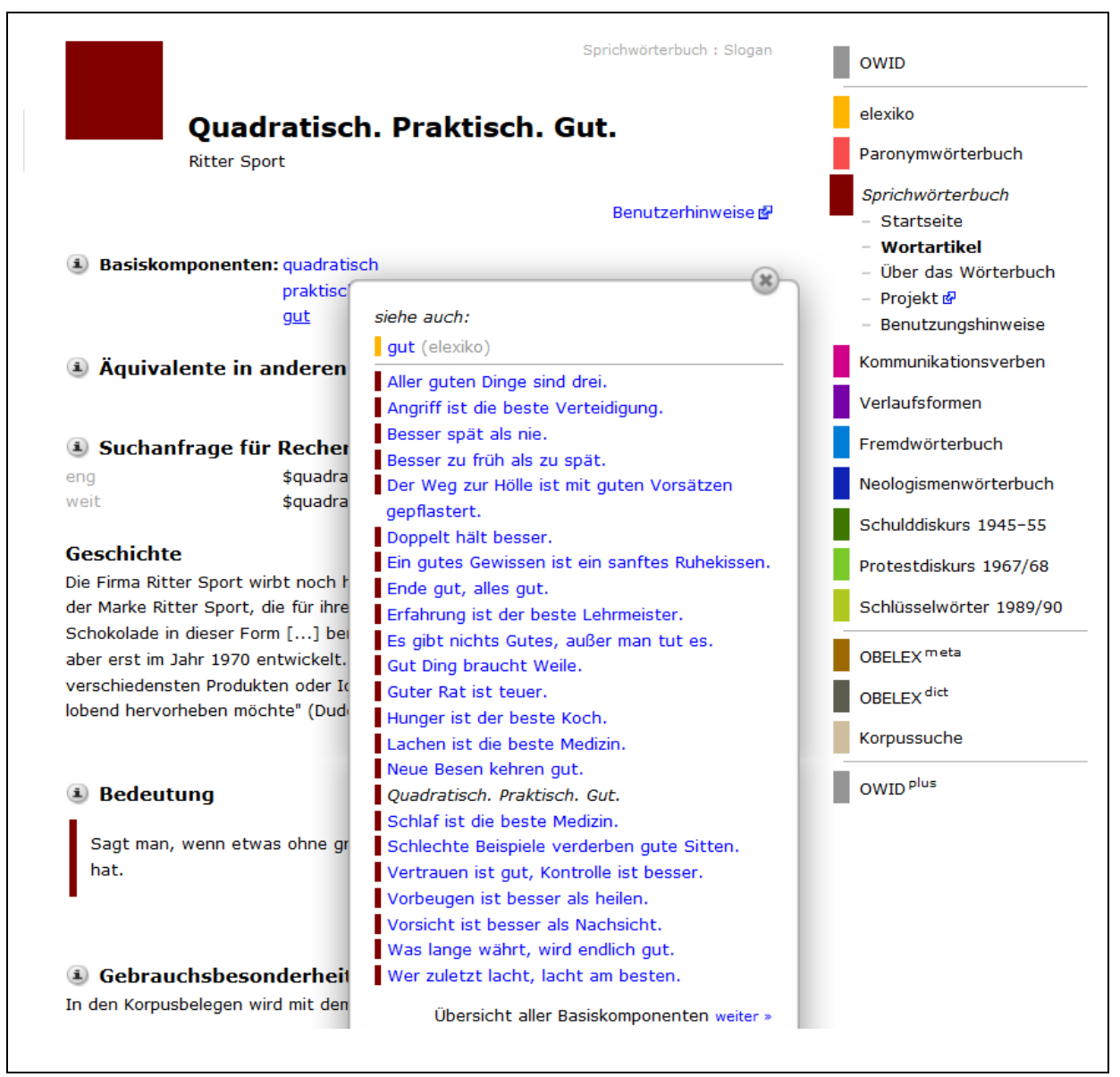

Abb. 12: Liste der Basiskomponenten (im Artikel Quadratisch auf $g u t)$

OWID sieht schließlich die Möglichkeit vor, unterschiedliche Stichwortlisten anzeigen zu lassen. So kann man den Slogan Da weiß man, was man hat als Teil des Sprichwörterbuchs sehen. Es ist aber auch möglich, die gesamte OWIDStichwortliste abzrufen.

\section{Schlussbemerkungen und Ausblick}

Die dargestellte lexikographische Behandlung von Werbeslogans im Rahmen von OWID ist (in der germanistischen Lexikographie) ein Novum und trägt zur lexikographischen Behandlung von polylexikalen Lexikon-Einheiten bedeutend bei; vor allem aus der Sicht der neueren Betrachtungen des Lexikons aus der Perspektive der Konstruktionsgrammatik. Mit dem hier vorge- 
stellten lexikografischen Prozess, der zum Modul Werbeslogans führte, verfolgten wir das Ziel, den Korpusgebrauch von aktuell gebräuchlichen deutschen Werbeslogans nach Kriterien der wissenschaftlichen Lexikografie aufzubereiten und als Onlinedokumentation nachhaltig zur Verfügung zu stellen. Da systematische korpusbasierte (Online-)Dokumentationen von Werbeslogans bis dato fehlen, dürfte das beschriebene Modul ein innovatives Konzept zur lexikografischen Erfassung und linguistischen Beschreibung der Werbeslogans darstellen. Anhand zahlreicher Korpusbelege konnte veranschaulicht werden, wie vielfältig und variabel der Gebrauch von Werbeslogans als verfestigten Sätzen in außerwerblichen Kontexten ist sowie wie und wozu Sprecher/-innen sie verwenden. Dadurch versuchten wir nicht nur $\mathrm{zu}$ verdeutlichen, dass Werbeslogans in der Gemeinsprache ähnlich wie Sprichwörter funktionieren und verwendet werden, sondern dass die Werbesprache eine moderne Quelle für die Entstehung neuer Sprichwörter darstellt. Es kann beobachtet werden, dass es in der Sprachgemeinschaft ein ausgeprägtes Bewusstsein von Sätzen als funktional vollständigen Einheiten gibt. Ob diese satzwertigen Gefüge als Sprichwort, Slogan, Motto, Devise u. a. bezeichnet werden, scheint für die Alltagssprecher/-innen unwichtig, was metakommunikative Elemente im Kotext von Slogans sowie Sprichwörtern veranschaulichen. Damit ließ sich auch die lexikografische Beschreibung von Werbeslogans nach demselben Modell wie dem für Sprichwörter sowie ihre Integration in das OWID-Sprichwörterbuch begründen. Stellenweise wurden die Slogan-Artikel jedoch im Hinblick auf die Spezifik der Werbeslogans erweitert („Kernform“, „Äquivalente in anderen Sprachen“, "Geschichte", „Vorkommen in Nachschlagewerken"), um auf die für Slogans wichtige Entstehungsgeschichte und ihre bisherige sporadische Dokumentation zu verweisen. Der künftige Schwerpunkt bei der lexikografischen Beschreibung des Korpusgebrauchs von Werbeslogans wird neben der Erarbeitung neuer Artikel auf der Weiterentwicklung der Methode der Erfassung lexikalischer Variationsmuster und von neuen Darstellungsformaten (z. B. in Form von Lückenfüllertabellen vgl. Steyer 2013) mit lexpan liegen.

Das OWID-Sprichwörterbuchmodul Werbeslogans richtet sich zum einen an In- und Auslandsgermanist/-innen und Deutschlehrende, die das Modul in ihre Lehrtätigkeit integrieren könnten, sowie an alle Deutschlernenden und Interessierten, die anhand zahlreicher Links das Thema Korpusgebrauch von Werbeslogans für sich entdecken wollen. Die korpusinformierte Untersuchung und Beschreibung von Werbeslogans soll aber auch für die Forschung anregend sein, insbesondere im Bereich der Sprichwortforschung und der Konstruktionsgrammatik, aber auch im Bereich der Werbesprachenforschung.

\section{Endnoten}

1. Es war die korpuslinguistische Wende, die das Erforschen und lexikografische Erfassen von sprachlichen Phänomenen im Allgemeinen und von Phraseologismen bzw. „usuellen Wortverbindungen" (Steyer 2013) im Speziellen tiefgreifend veränderte. Die korpuslinguistische 
Wende führte im Bereich der Phraseologie zu zwei Paradigmenwechsen: Liberalisierung und Erweiterung der phraseologischen Einheiten in Richtung "funktionale Verfestigung" und musterbasierte Phraseologie (vgl. Steyer 2013).

2. Das Wörterbuchportal OWID enthält neben dem Sprichwörterbuch folgende lexikografische Ressourcen: elexiko - Online-Wörterbuch zur deutschen Gegenwartssprache (online seit 2013), Paronymwörterbuch (online seit 2018), Kommunikationsverben (online seit 2013), Kleines Wörterbuch der Verlaufsformen im Deutschen (online seit 2013), Deutsches Fremdwörterbuch - Neubearbeitung (Buchstaben A-H online seit 2016), Neologismenwörterbuch (online seit 2014), Schulddiskurs 1945-55 (online seit 2008), Protestdiskurs 1967/68 (online seit 2012), Schlüsselwörter der Wendezeit 1989/90 (online seit 2015).

3. Die deutschen Wörterbuchartikel wurden im Rahmen des multilingualen EU-Projekts SprichWort. Eine Internetplatform für das Sprachenlernen (2008-2010) erarbeitet und anschließend von Dr. Kathrin Steyer für die Online-Publikation zubereitet.

4. Bekannte Werbeslogans wurden beispielsweise im Slowenischen noch seltener lexikografisch erfasst: Einige Slogan-Artikel finden sich in einer kolaborativ geschriebenen OnlineRessource des gesprochenen Slowenisch „Razvezani jezik“ (http://razvezanijezik.org/ ?page=Naslovnica), die punktuell die Entstehungsgeschichte und die Bedeutung skizzieren, ohne konkrete Sprachbelege anzuführen. Weitere lexikografische Ressourcen in anderen Sprachen sind mir nicht bekannt.

5. Wie in jüngsten korpusempirischen Arbeiten im Bereich der Phraseologie üblich, wird auch in der vorliegenden Untersuchung eine vordefinierte Liste von Slogans mit vermuteter Tendenz zur Usualisierung anhand eines virtuellen Korpus aus DEREKO validiert. Allerdings wird auch bei einem korpusbasierten („,corpus-based“) Vorgehen wie diesem immer wieder beobachtet, dass Erkenntnisse anhand Korpusevidenzen in den Forschungsprozess einfließen und ihn beeinflussen. Folglich wird die Festlegung auf ein Korpusparadigma in jüngster Zeit von Forschern relativiert: Der corpus-based- und corpus-driven-Ansatz schließen sich gegenseitig nicht aus und nur ihre Verknüpfung kann wirklich ertragreich sein (vgl. Steyer 2013: 71-72). Um dieser Relativierung terminologisch gerecht zu werden, wird in der vorliegenden Untersuchung in Anlehnung an Gredel (2014) von einem korpusinformierten Ansatz gesprochen.

6. Die Adaption der musterbasierten Korpusmethodlogie für Slogans und ihre lexikografische Beschreibung im Modul Werbeslogans im OWID-Sprichwörterbuch wurden in Kooperation mit dem Projekt Usuelle Wortverbindungen (Leitung: Dr. Kathrin Steyer) während mehrerer Forschungsaufenthalte seit 2009 am Leibniz-Institut für Deutsche Sprache erarbeitet.

7. Die Suchprozedur basiert auf einer bestimmten Suchsyntax, dessen Komplexität von der Komplexität der Oberflächenform des Slogans abhängt. Diese wurde im Rahmen des EUProjekts SprichWort. Eine Internetplattform für das Sprachenlernen sowie des UWV-Projekts entwickelt.

8. Ergebnisse der Suchanfragen werden zunächst in Form von KWIC-Ansicht (Kontextzeilen des Suchwortes/des Suchsyntagmas) analysiert. Die KWIC-Ansicht liefert einen ersten Überblick dazu, ob die Suchanfrage bereits eine hohe Anzahl an relevanten Treffern erbringt oder ob sie durch bestimmte Parameter anzupassen ist, d. h. ob die Suchanfrage zu eng oder zu weit ist. $\mathrm{Zu}$ jeder KWIC-Zeile kann man einen Volltext abrufen, der je nach Wahl einige (Ab-)Sätze vor und nach dem zentralen Suchsyntagma angibt. 
9. Das Analysewerkzeug wurde vom Projekt Usuelle Wortverbindungen (UWV) am LeibnizInstitut für Deutsche Sprache in Mannheim entwickelt.

10. Bevor mit dem Analyseprogramm lexpan eine KWIC-Systematisierung erfolgen kann, müssen zunächst anhand einer adäquaten Suchanfrage aus dem DEREKO entsprechende KWIC-Listen ermittelt werden. Diese werden in lexpan exportiert und darin abgespeichert sowie in einem nächsten Schritt mithilfe von einfachen Suchanfragen ohne komplexe Suchsyntax systematisch und schnell untersucht.

\section{Literaturverzeichnis}

Androutsopoulos, Jannis. 1997. Intertextualität in jugendkulturellen Textsorten. Klein, Josef und Ulla Fix (Eds.). 1997. Textbeziehungen. Linguistische und literaturwissenschaftliche Beiträge zur Intertextualität: 339-372. Tübingen: Stauffenburg.

Betz, Ruth. 2006. Gesprochensprachliche Elemente in deutschen Zeitungen. Radolfzell: Verlag für Gesprächsforschung.

Bubenhofer, Noah. 2009. Sprachgebrauchsmuster. Korpuslinguistik als Methode der Diskurs- und Kulturanalyse. Sprache und Wissen 4. Berlin/New York: Walter de Gruyter.

Büchmann, Georg. 1864, 422001. Geflügelte Worte: der klassische Zitatenschatz. 42. neu bearb. u. aktual. Aufl. München: Ullstein Verlag.

Burger, Harald. 2015. Phraseologie. Eine Einführung am Beispiel des Deutschen. 5. Aufl. Berlin: Erich Schmidt Verlag.

Burger, Harald, Annelies Buhofer und Ambros Sialm. 1982. Handbuch der Phraseologie. Berlin/ New York: Walter de Gruyter.

Cosmas-II (Projekt „Cosmas II“ (2015). Korpusrecherche- und -analysesystem. Institut für Deutsche Sprache. Mannheim). http://www.ids-mannheim.de/cosmas2/ [12.1.2021].

DEREKO (Institut für Deutsche Sprache (2016): Deutsches Referenzkorpus / Archiv der Korpora geschriebener Gegenwartssprache 2016-I (Release vom 31.03.2016)). Mannheim: LeibnizInstitut für Deutsche Sprache. www.ids-mannheim.de/DeReKo [12.1.2021].

Duden 12: Zitate und Aussprïche. 2002, ${ }^{32008 . ~ M a n n h e i m / L e i p z i g / W i e n / Z u ̈ r i c h: ~ D u d e n v e r l a g . ~}$

Ďurčo, Peter, Kathrin Steyer und Katrin Hein. 2017. Sprichwörter im Gebrauch. Unveränderter Wiederabdruck der 2015 in Trnava erschienenen Erstausgabe. Mannheim: Institut für Deutsche Sprache.

elexiko (Online-Wörterbuch zur deutschen Gegenwartssprache). http://www.owid.de/wb/elexiko/start. html [12.1.2021].

Engelberg, Stefan, Annette Klosa-Kückelhaus und Carolin Müller-Spitzer. 2019. Lexikographie zwischen Grimm und Google? Sprachreport 35(2): 30-34.

Fix, Ulla. 1997. Kanon und Auflösung des Kanons. Typologische Intertextualität - ein ,postmodernes' Stilmittel? Eine thesenhafte Darstellung. Antos, Gerd und Heike Tietz (Eds.). 1997. Die Zukunft der Textlinguistik. Traditionen, Transformationen, Trends: 97-108. Reihe Germanistische Linguistik 188. Tübingen: Niemeyer.

Fix, Ulla. 2007. Der Spruch - Slogans und andere Spruchtextsorten. Burger, Harald et al. (Eds.). 2007. Phraseologie. Ein internationales Handbuch der zeitgenössischen Forschung: 1. Halbband: 459-468. Handbücher für Sprach- und Kommunikationswissenschaft 28(1). Berlin/New York: Walter de Gruyter. 
Gredel, Eva. 2014. Diskursdynamiken. Metaphorische Muster zum Diskursobjekt Virus. Berlin: Mouton de Gruyter.

Hars, Wolfgang. 2002. Das Lexikon der Werbesprüche. 500 bekannte deutsche Werbeslogans und ihre Geschichte. Frankfurt a. Main: Piper Verlag.

Hemmi, Andrea. 1994. „Es muß wirksam werben, wer will nicht verderben“: Kontrastive Analyse von Phraseologismen in Anzeigen-, Radio- und Fernsehwerbung. Zürcher Germanistische Studien. Frankfurt a. M.: Peter Lang.

Janich, Nina. 1997. Wenn Werbung mit Werbung Werbung macht ... Ein Beitrag zur Intertextualität. Muttersprache 107: 297-309.

Janich, Nina. 2010. Werbesprache. Ein Arbeitsbuch. 5. erw. Aufl. Tübingen: Narr Studienbücher.

Janich, Nina. 2019. Intertextualität und Text(sorten)vernetzung. Janich, Nina (Ed.). 2019. Textlinguistik. 15 Einführungen und eine Diskussion. 2. Aufl. 177-198. Tübingen: Narr.

Jeromin, Rolf. 1969 (о. J.). Zitatenschatz der Werbung. Slogans erobern Märkte. Gütersloh: Präsentverlag Heinz Peter.

Jesenšek, Vida. 2011. Sprichwörter im Wörterbuch. Linguistik online 47(3): 67-78. http://www. linguistik-online.de/47_11/.

Jesenšek, Vida. 2013. Das lexikographische Beispiel in der Parömiographie. Formen und Funktionen. Lexikos 23: 150-171. http://lexikos.journals.ac.za/pub/article/view/1209/720.

Keibel, Holger. 2008. Mathematische Häufigkeitsmaße in der Korpuslinguistik: Eigenschaften und Verwendung. Mannheim: Institut für Deutsche Sprache. URL: http://www.ids-mannheim.de/kl/ dokumente/freqMeasures.html [13.07.2020].

Klosa, Annette und Carolin Müller-Spitzer unter Mitarbeit von Martin Loder. 2016. Internetlexikografie. Ein Kompendium. Berlin/Boston: Walter de Gruyter.

Klosa-Kückelhaus, Annette und Carolin Müller-Spitzer. 2019. OWID und OWIDplus: lexikographische und lexikalische Ressourcen am IDS Mannheim. Zeitschrift für germanistische Linguistik 47(2): 418-431. Berlin/Boston: Walter de Gruyter.

lexpan - Lexical Pattern Analyzer (Version 2019-06-21). Ein Analysewerkzeug zur Untersuchung syntagmatischer Strukturen auf der Basis von Korpusdaten. Entwickelt vom Projekt "Usuelle Wortverbindungen", Institut für Deutsche Sprache, Mannheim. http://uwv.ids-mannheim. de/lexpan/ [13.1.2020].

Liste geflügelter Worte. https://de.wikipedia.org/wiki/Liste_gefl\%C3\%BCgelter_Worte [12.1.2020].

Lüger, Heinz-Helmut. 1998. Vom Zitat zur Adaption. Zu einigen Verwendungsweisen satzwertiger Phraseologismen. Beiträge zur Fremdsprachenvermittlung 34: 118-135.

Lüger, Heinz-Helmut. 1999. Satzwertige Phraseologismen. Eine pragmalinguistische Untersuchung. Wien: Ed. Praesens.

OWID (Online-Wortschatz-Informationssystem Deutsch des IDS). https://www.owid.de/ [12.1.2021].

Polajnar, Janja. 2012. Textuelle Aspekte von rekontextualisierten Werbeslogans in deutschsprachigen Zeitungen. Eine korpusbasierte Untersuchung bekannter Werbeslogans im Zeitungskorpus des Deutschen Referenzkorpus (DeReKo). Muttersprache 122(1): 48-64.

Polajnar, Janja. 2016. Recontextualisation of International Advertising Slogans and their Equivalents in Different European Languages. Poznań Studies in Contemporary Linguistics [Online ed.] 52(1): 85-117. 
Polajnar, Janja. 2019. Werbeslogans im aktuellen Sprachgebrauch. Eine korpusinformierte, diachrone Untersuchung zur Dynamik des Slogan-Gebrauchs mit lexikografischen Fallstudien. Amades 55. Mannheim: IdS Leibniz-Institut für Deutsche Sprache.

Razvezani jezik. Prosti slovar žive slovenččine. http://razvezanijezik.org/?page=Naslovnica [12.3.2021] Redensarten-Index (Wörterbuch für Redensarten, Redewendungen, idiomatische Ausdrücke und feste Wortverbindungen). http://www.redensarten-index.de/suche.php [12.1.2021].

Schlobinski, Peter. 1989. Frau Meier hat Aids, Herr Tropfmann hat Herpes, was wollen Sie einsetzen? Exemplarische Analyse eines Sprechstils. Schlobinski, Peter et al. (Eds.). 1989. OBST 16. Sprache und Erfahrung: 1-34. Osnabrücker Beiträge zur Sprachtheorie 41. Osnabrück: Univ. Osnabrück.

Slogans.de. http://www.slogans.de/slogans.php?Op=SRanking1 [12.1.2021].

Steyer, Kathrin (Ed.). 2012. Sprichwörter multilingual. Theoretische, empirische und angewandte Aspekte der modernen Parömiologie. Studien zur Deutschen Sprache 60. Tübingen: Gunter Narr Verlag.

Steyer, Kathrin. 2013. Usuelle Wortverbindungen. Zentrale Muster des Sprachgebrauchs aus korpusanalytischer Sicht. Studien zur Deutschen Sprache 65. Tübingen: Narr.

Steyer, Kathrin (Hg.). 2018. Sprachliche Verfestigung. Wortverbindungen, Muster, Phrasem-Konstruktionen. Studien zur Deutschen Sprache 79. Tübingen: Narr.

Steyer, Kathrin und Peter Duurčo. 2013. Ein korpusbasiertes Beschreibungsmodell für die elektronische Sprichwortlexikografie. Benayoun, Jean-Michel, Natalie Kübler und Jean-Philippe Zouogbo (Eds.). 2013. Parémiologie. Proverbes et formes voisines. Band 3: 219-250. Sainte Gemme: PUSG.

Steyer, Kathrin und Janja Polajnar. 2015. Werbeslogans. Sprichwörterbuch in OWID. http://www. owid.de/wb/sprw/start.html.

SWB: Sprichwörterbuch in OWID. http://www.owid.de/wb/sprw/start.html [12.1.2020].

SWB-SL: Steyer, Kathrin und Janja Polajnar. 2015. Modul Werbeslogans im OWID-Sprichwörterbuch. https://www.owid.de/service/stichwortlisten/slgn [12.1.2020].

SWP (EU-Sprichwortplattform). http://www.sprichwort-plattform.org/ [12.1.2020]. 